Article

\title{
Dietary Polysaccharide from Enteromorpha Clathrata Modulates Gut Microbiota and Promotes the Growth of Akkermansia muciniphila, Bifidobacterium spp. and Lactobacillus spp.
}

\author{
Qingsen Shang ${ }^{1}$, Ya Wang ${ }^{1}$, Lin Pan ${ }^{1}$, Qingfeng Niu ${ }^{1}$, Chao Li ${ }^{1}$, Hao Jiang ${ }^{1,2}$, Chao Cai ${ }^{1,2}$, \\ Jiejie Hao ${ }^{1,2}$, Guoyun $\mathrm{Li}^{1,2}$ and Guangli Yu ${ }^{1,2, *(1)}$ \\ 1 Key Laboratory of Marine Drugs of Ministry of Education, and Shandong Provincial Key Laboratory of \\ Glycoscience and Glycotechnology, School of Medicine and Pharmacy, Ocean University of China, \\ Qingdao 266003, China; shangqingsen@163.com (Q.S.); 15222175867@163.com (Y.W.); \\ pl_panlin@163.com (L.P.); qfengniu@163.com (Q.N.); lichaolbj@163.com(C.L.); haojiang833@163.com (H.J.); \\ caic@ouc.edu.cn (C.C.); 2009haojie@ouc.edu.cn (J.H.); liguoyun@ouc.edu.cn (G.L.) \\ 2 Laboratory for Marine Drugs and Bioproducts, Qingdao National Laboratory for Marine Science and \\ Technology, Qingdao 266003, China \\ * Correspondence: glyu@ouc.edu.cn; Tel.: +86-532-8203-1609
}

Received: 24 April 2018; Accepted: 15 May 2018; Published: 17 May 2018

\begin{abstract}
Recently, accumulating evidence has suggested that Enteromorpha clathrata polysaccharide (ECP) could contribute to the treatment of diseases. However, as a promising candidate for marine drug development, although ECP has been extensively studied, less consideration has been given to exploring its effect on gut microbiota. In this light, given the critical role of gut microbiota in health and disease, we investigated here the effect of ECP on gut microbiota using 16S rRNA high-throughput sequencing. As revealed by bioinformatic analyses, ECP considerably changed the structure of the gut microbiota and significantly promoted the growth of probiotic bacteria in C57BL/6J mice. However, interestingly, ECP exerted different effects on male and female microbiota. In females, ECP increased the abundances of Bifidobacterium spp. and Akkermansia muciniphila, a next-generation probiotic bacterium, whereas in males, ECP increased the population of Lactobacillus spp. Moreover, by shaping a more balanced structure of the microbiota, ECP remarkably reduced the antigen load from the gut in females. Altogether, our study demonstrates for the first time a prebiotic effect of ECP on gut microbiota and forms the basis for the development of ECP as a novel gut microbiota modulator for health promotion and disease management.
\end{abstract}

Keywords: Enteromorpha clathrata; polysaccharide; gut microbiota; prebiotic; Bifidobacterium spp.; Akkermansia muciniphila; Lactobacillus spp.

\section{Introduction}

Enteromorpha clathrata is an edible green alga that has been traditionally consumed as a folk medicine and a natural herb in Asian countries for the treatment of inflammation-associated diseases [1,2]. Recently, a significant body of literature has demonstrated that polysaccharide from E. clathrata possesses numerous bioactivities including, but not limited to, anticoagulative [3], immunomodulatory [4], antioxidant [2,5], anticancer [6] and antiobesity [1,2]. Since seaweed polysaccharides are among the focus of marine biomedical research today $[7,8]$, these discoveries make E. clathrata polysaccharide (ECP) an excellent candidate for marine drug development. However, to date, although different bioactivities of ECP have been studied, its effect on gut microbiota has not been explored. 
Gut microbiota is an "invisible organ" that has been discovered to play pivotal roles in health and disease $[9,10]$. In recent years, it has become increasingly clear that disruption of the gut microbiota, which is commonly termed as "gut dysbiosis", is robustly associated with various diseases and disorders such as diabetes [11], obesity [11,12], hypertension [13], colorectal cancer [14], multiple sclerosis [15], asthma [16], autism [17], Alzheimer's disease [18], Parkinson's disease [19] and non-alcoholic fatty liver disease [20]. Recent findings suggest an effective strategy for the management of diseases by targeting the gut microbiota $[9,10,21]$, and among the commercially available therapeutics that are clinically used to treat dysbiosis and cure diseases, prebiotic stands out as a very effective one [22-24]. Previous studies have indicated that seaweed polysaccharides constitute an appreciable proportion of bioactive compounds for prebiotic development $[25,26]$. However, whether or not ECP has any prebiotic effects has not yet been determined.

In the present study, the dearth of previous research, coupled with the fact that ECP cannot be absorbed after oral intake in the gastrointestinal tract [26,27], prompts us to investigate what impact ECP has on gut microbiota and whether it could be used as a prebiotic. To these ends, we treated male and female C57BL/6J mice with high $(100 \mathrm{mg} / \mathrm{kg} /$ day $)$ and low $(50 \mathrm{mg} / \mathrm{kg} /$ day $)$ doses of ECP and studied its effect on gut microbiota using $16 \mathrm{~S}$ rRNA high-throughput sequencing. We found that ECP significantly changed the structure of the gut microbiota and exerted different prebiotic effects on Akkermansia muciniphila, Bifidobacterium spp. and Lactobacillus spp. in male and female mice. Our study provides the first proof-of-concept for the prebiotic effect of ECP and formed the basis for the development of ECP as a drug or a food supplement for health promotion and treatment of dysbiosis.

\section{Results and Discussion}

\subsection{Dietary ECP Changed the Structure and Increased the Richness and Diversity of the Gut Microbiota in Male and Female Mice}

Previous studies indicate that ECP could be degraded by specific microbes in the gut [26,27], however, the precise effect of ECP on the whole gut microbiota is unknown. Therefore, to address this issue, we investigated here in detail the in vivo effect of ECP on intestinal microorganisms using 16S rRNA high-throughput sequencing. As revealed by principal component analysis (PCA), both low and high doses of ECP significantly changed the structure of the gut microbiota in C57BL/6J mice (Figure 1). Besides, alterations of the gut microbiota were more obvious in male mice than in female mice (Figure 1). This suggests that ECP has a sex-specific effect on gut microbiota. Interestingly, preceding studies have also found similar effects of chondroitin sulfate [28] and keratan sulfate [29] on gut microbiota. Given that sex hormones play a critical role in determining the composition of the intestinal microorganisms [30], our results and past studies [28,29] highlight that dietary fibers may have a gender-specific effect on gut microbiota and clinical therapies using dietary fibers for the treatment of dysbiosis-associated diseases should be tailored according to individuals' sex.

Since ECP changed the structure of the gut microbiota, we then sought to investigate its effect on the richness and diversity of the intestinal microorganisms. To this aim, the values of the richness estimators, Chao1 and observed species, and the values of the diversity estimator, Shannon indices, were respectively calculated. Interestingly, ECP was found to significantly increase the richness and diversity of the gut microbiota in male and female mice (Figure 2).

It is of interest to note that in Figure 1A, PCA1 and PCA2 explained about $60 \%$ of the variance for the male microbiota, while in Figure 1B, PCA1 and PCA2 only explained about $50 \%$ of the variance for the female microbiota. As have detailed above, the structure of the male microbiota is quite different from that of the female microbiota. In addition, previous studies have also demonstrated that sex hormones play a critical role in determining the composition of the gut microbiome [30]. Therefore, it is reasonable that the PCA plot of the female microbiota and male microbiota were different. Another reason for the dissimilarities of the PCA plot might be due to the fact that compared to the male microbiota, the female microbiota is more reluctant to ECP treatment. This means that ECP 
induces much less alterations in the composition of the female microbiota than that of male microbiota. In addition, this can be evidenced by the Chao1 indices, observed species and Shannon indices. As shown in Figure 2, ECP treatment resulted in a much more dramatic increase in Chao1 indices, observed species and Shannon indices in the microbiota of male mice, while in contrast, in female mice, only the microbiota from the low-dosage group seemed to have increased values of Chao1 indices and Shannon indices. This is in quite accordance with the PCA plot analysis. In male microbiota, both low-dosage ECP and high-dosage ECP significantly changed the structure of the microbiota along the PCA1 axis, which explained $42.07 \%$ of the variance (Figure 1A). However, in female microbiota, only the low-dosage group had the most obvious alterations in the composition of the microbiota as it changed both along the PCA1 axis (explained $26.83 \%$ of the variance) and the PCA2 axis (explained $22.73 \%$ of the variance) (Figure 1B). Although treatment of high-dosage ECP also modulated the composition of the microbiota in the FH group, it changed largely only along the PCA1 axis (explained $26.83 \%$ of the variance). This also explains why the compositions of the microbiota in FN group and FH group are closely related in comparison to the FL group.
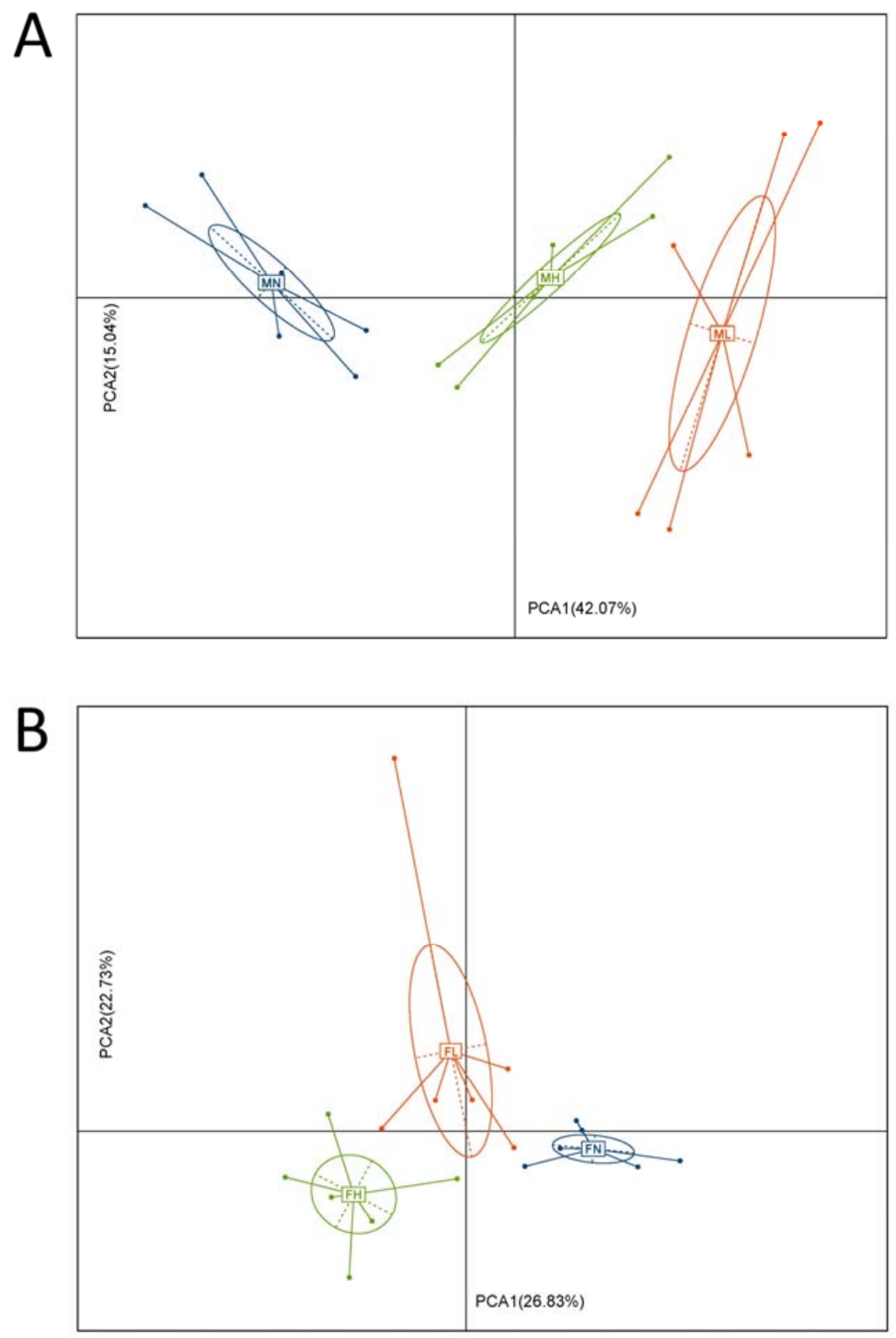

Figure 1. Administration of ECP significantly changed the structure of the gut microbiota. PCA score plot of the first and second components for the gut microbiome in male (A) and female mice (B). Blue indicates control groups, red indicates low dose groups and green indicates high dose groups. The PCA score plot was constructed based on the OTUs of the microbiota in male mice and female mice. 


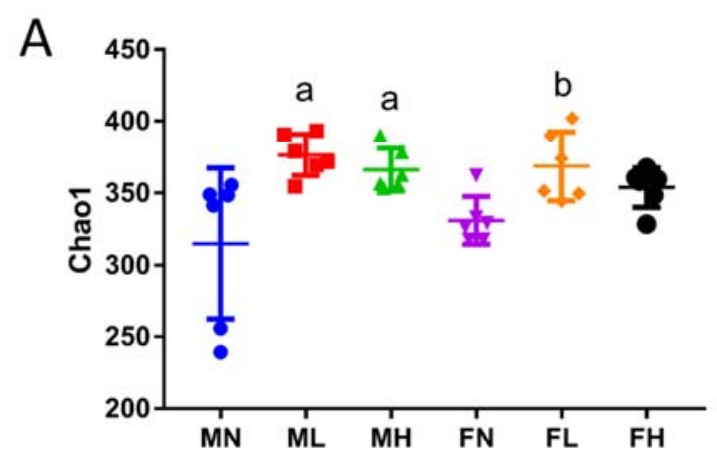

B

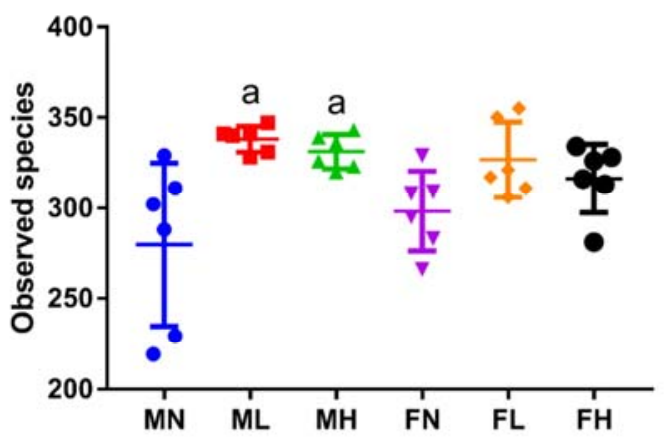

C

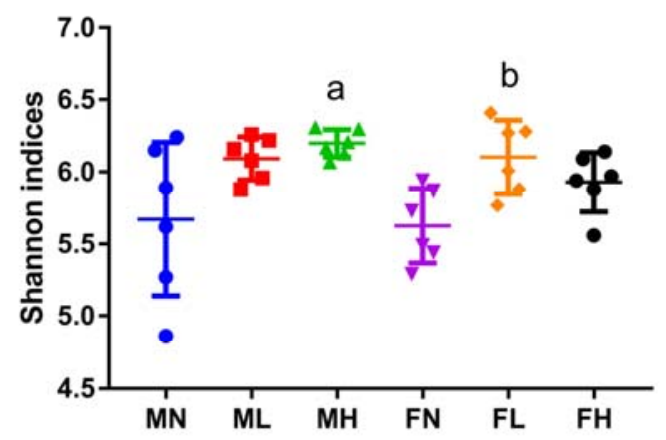

Figure 2. Dietary ECP increased the richness and diversity of the gut microbiota. Chao1 (A) and observed species (B) were used as the richness estimators. Shannon indices (C) was used as the diversity estimator. a: $p<0.05$ vs. MN group; b: $p<0.05$ vs. FN group.

Previous studies indicate that a fiber-deficient diet could decrease the richness and diversity of the gut microbiome and increase the susceptibility of the host to pathogen infection and intestinal diseases [31]. Besides, mounting evidence suggests that supplementation of dietary fiber or microbiota-accessible carbohydrate to the daily diet could help to maintain intestinal symbiosis and reduce the incidence of bacterial colonization [32,33]. In line with these results, our study demonstrated that ECP as a dietary fiber could modify the composition of the gut microbiota and increase species diversity and richness. Our findings support the use of ECP as a novel gut microbiota modulator and encourage continued investigation on the beneficial effect of ECP on gut microbiota in mouse models of dysbiosis-associated diseases.

2.2. Dietary ECP Modulated the Intestinal Microbiota at Different Taxonomic Levels and Proundly Increased the Populations of Akkermansia muciniphila, Bifidobacterium spp. and Lactobacillus spp.

Given that ECP could change the structure of the microbiota, we next asked what impact ECP has on the abundance of each bacterium in the gut. We compared the bacterial composition of the gut microbiota at the phylum level and found that ECP significantly increased the abundance of Bacteroidetes and decreased the population of Firmicutes in male mice (Figure 3A). However, interestingly, in female mice, ECP decreased the amount of Bacteroidetes and increased the proportion of Firmicutes (Figure 3A). Besides, ECP was also found to has a differing effect on the abundance of Proteobacteria in male and female mice. Male mice treated with ECP had increased levels of Proteobacteria while female mice fed with ECP had reduced levels of Proteobacteria (Figure 3A). 


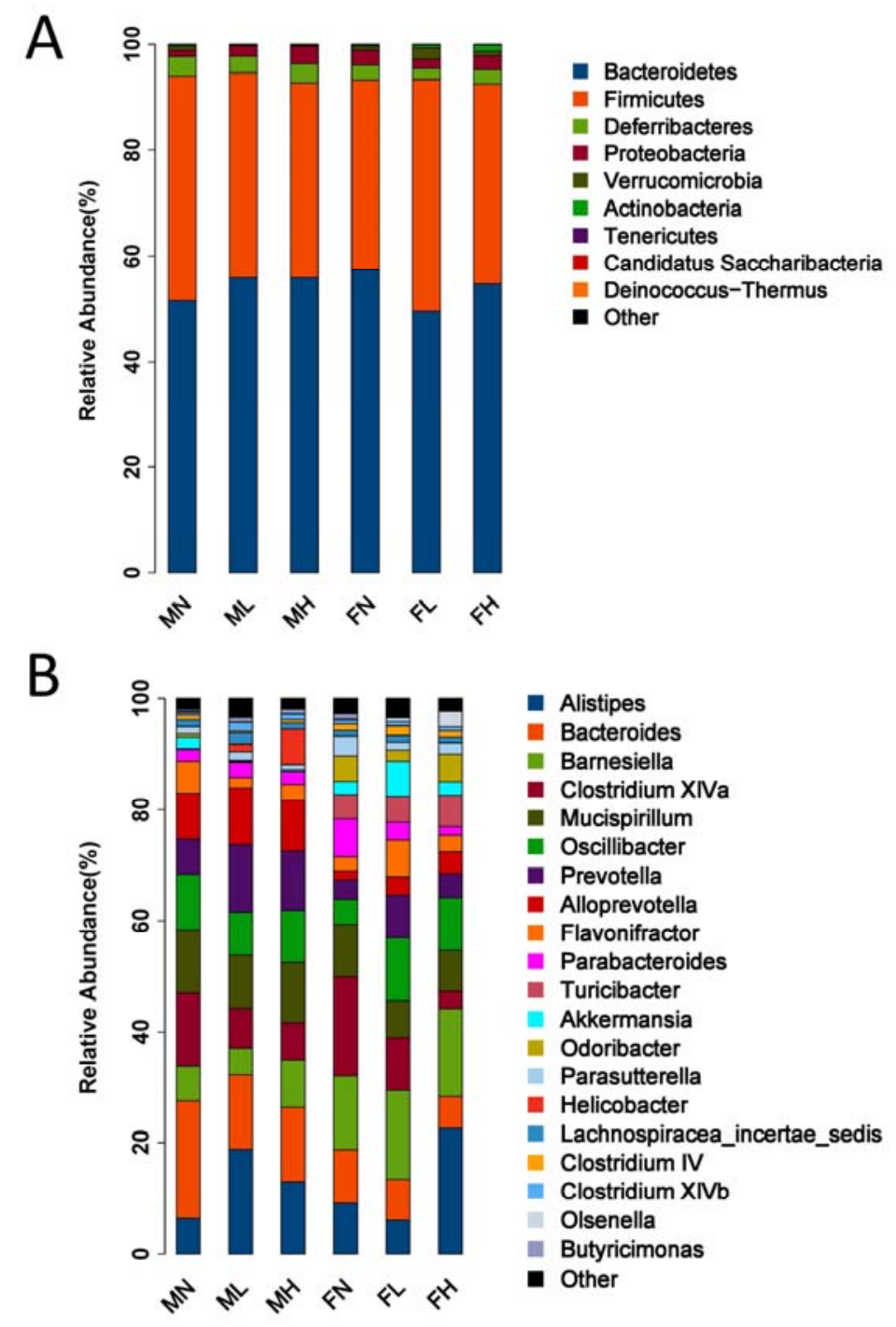

Figure 3. Response of the gut microbiota to ECP treatment at the phylum (A) and genus (B) levels. The relative abundances of the gut bacteria presented here were calculated by averaging the data obtained from the six replicates within each group.

We further compared the bacterial composition of the intestinal microbiota at the genus level and found similar divergent effects of ECP on the abundances of Alistipes spp., Oscillibacter spp., Flavonifractor spp., Parabacteroides spp. and Akkermansia spp. in male and female mice (Figure 3B). For example, in male mice, ECP decreased the population of Oscillibacter spp., while in contrast, in female mice, ECP increased that of Oscillibacter spp. Collectively, these findings demonstrate that the male microbiota and female microbiota respond oppositely to ECP treatment, which, in accordance with the PCA results, provides a further proof of the sex-specific effect of ECP on gut microbiome.

To fully elucidate the effect of ECP on gut microbiota, we performed the linear discriminant analysis (LDA) effect size (LEfSe) analysis. The taxonomic cladogram and LDA score obtained from LEfSe analysis confirmed and visualized the modulatory effect of ECP on gut microbiota (Figures 4 and 5). Briefly, in male mice, ECP increased the abundances of Bacteroides spp., Prevotella spp., Alloprevotella spp., Butyricimonas spp., Eubacterium spp., and Peptococcus spp. and decreased the proportion of Helicobacter spp., while in contrast, in female mice, ECP increased the abundances of Odoribacter spp. Clostridium IV, Oscillibacter spp. and Alistipes spp. and decreased the proportions of 
Betaproteobacteria and Parabacteroides spp. (Figures 4 and 5). These observations are consistent with the sex-dependent effect of ECP on gut microbiota.

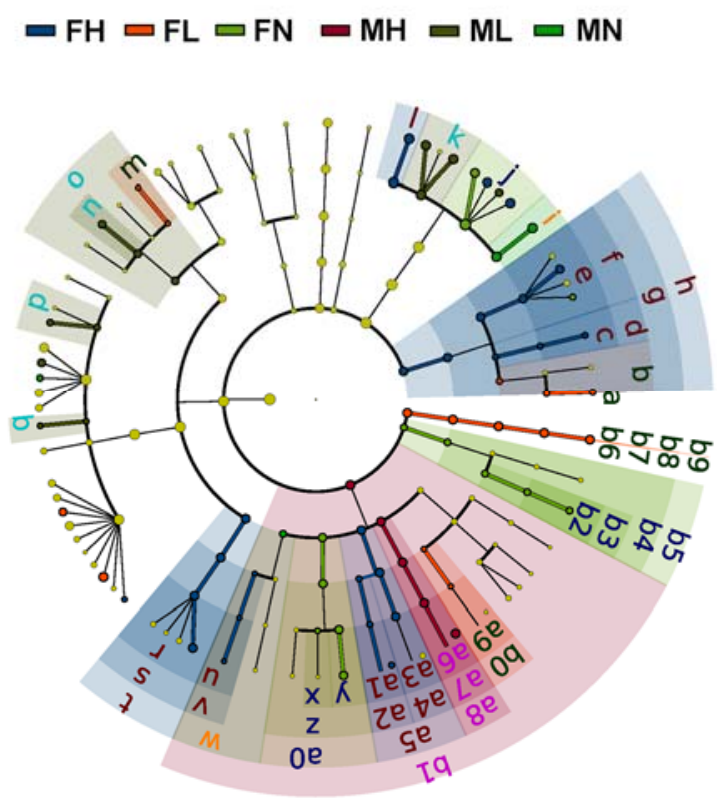

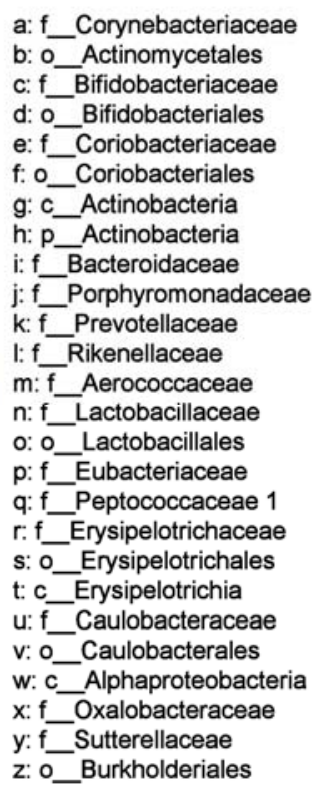

a: Corynebacteriacea

c: f_-Bifidobacteriaceae

d: o_Bifidobacteriales

eriobacteriacea

f. O_Coriobacteriales

h. p Actinobacteria

i: $f$ Bacteroidaceae

j: f__Porphyromonadaceae

k. f Prevotellaceae

n: $f$ Lactobacillacea

o: o Lactobacillales

p: f Eubacteriaceae

if - Erysipelotrichace

s: o Erysipelotrichales

t: c_Erysipelotrichia

Caulobacteracea

w: c_Alphaproteobacteria

y: f Sutterellaceae

z: o_Burkholderiales

\author{
a0: c_Betaproteobacteria \\ a1: f_Bdellovibrionaceae \\ a2: o Bdellovibrionales \\ a3: f_Desulfovibrionaceae \\ a4: O_Desulfovibrionales \\ a5: c Deltaproteobacteria \\ a6: f__Helicobacteraceae \\ a7: o_Campylobacterales \\ a8:c Epsilonproteobacteria \\ a9: f__Aeromonadaceae \\ b0: o_Aeromonadales \\ b1: p_Proteobacteria \\ b2: $f$ Anaeroplasmataceae \\ b3: - __Anaeroplasmatales \\ b4: c__Mollicutes \\ b5: $\mathrm{p}$ Tenericutes \\ b6: f_-Verrucomicrobiaceae \\ b7: o_Verrucomicrobiales \\ b8: c__Verrucomicrobiae \\ b9: p_Verrucomicrobia
}

Figure 4. The taxonomic cladogram obtained from LEfSe analysis of gut microbiota in different groups. The microbial compositions of the male and female mice were compared at different evolutionary levels. A significant value of less than 0.05 was used as a threshold for the LEfSe analysis.

Intriguingly, LEfSe analysis also identified that the abundances of the probiotic bacteria, including Lactobacillus spp., Bifidobacterium spp. and Akkermansia spp., were significantly different between the ECP-treated groups and control groups (Figures 4 and 5). By comparing in detail the populations of the probiotic bacteria in all the six groups, we found that ECP significantly increased the abundances of Bifidobacterium spp. and Akkermansia spp. in female mice and that of Lactobacillus spp. in male mice (Figure 6). Specifically, ECP increased the amount of Lactobacillus spp. by about 35-fold in the ML group and by about 8-fold in the MH group (Figure 6). Similarly, ECP increased the amount of Bifidobacterium spp. by about 2-fold in the FL group and by about 3-fold in the FH group (Figure 6). Besides, in the FL group, ECP also significantly increased the population of Akkermansia spp. by about 3-fold (Figure 6). Collectively, these observations demonstrated a beneficial prebiotic effect of ECP on the gut microbiota in male and female mice.

Short chain fatty acids (SCFAs) are a class of critically important bacterial metabolites, which are produced by specific intestinal bacteria during fermentation of dietary fibers in the gut [34-36]. Accumulating evidence from animal and human studies suggests that SCFAs play a beneficial role in modulating host physiology, and increasing the concentrations of SCFAs in the gut contributes to the treatment of dysbiosis-associated diseases [37-39]. Here we discovered that the abundances of SCFA-producing bacteria [34-36], including Bacteroides spp., Prevotella spp., Alloprevotella spp., Butyricimonas spp., Eubacterium spp., Odoribacter spp. and Clostridium IV were significantly increased in the gut microbiota of male and female mice. Given the critical role of SCFAs in modulating host metabolism and physiology [37-39], these data suggest a favorable effect of ECP on the gut microbiota of C57BL/6J mice. 


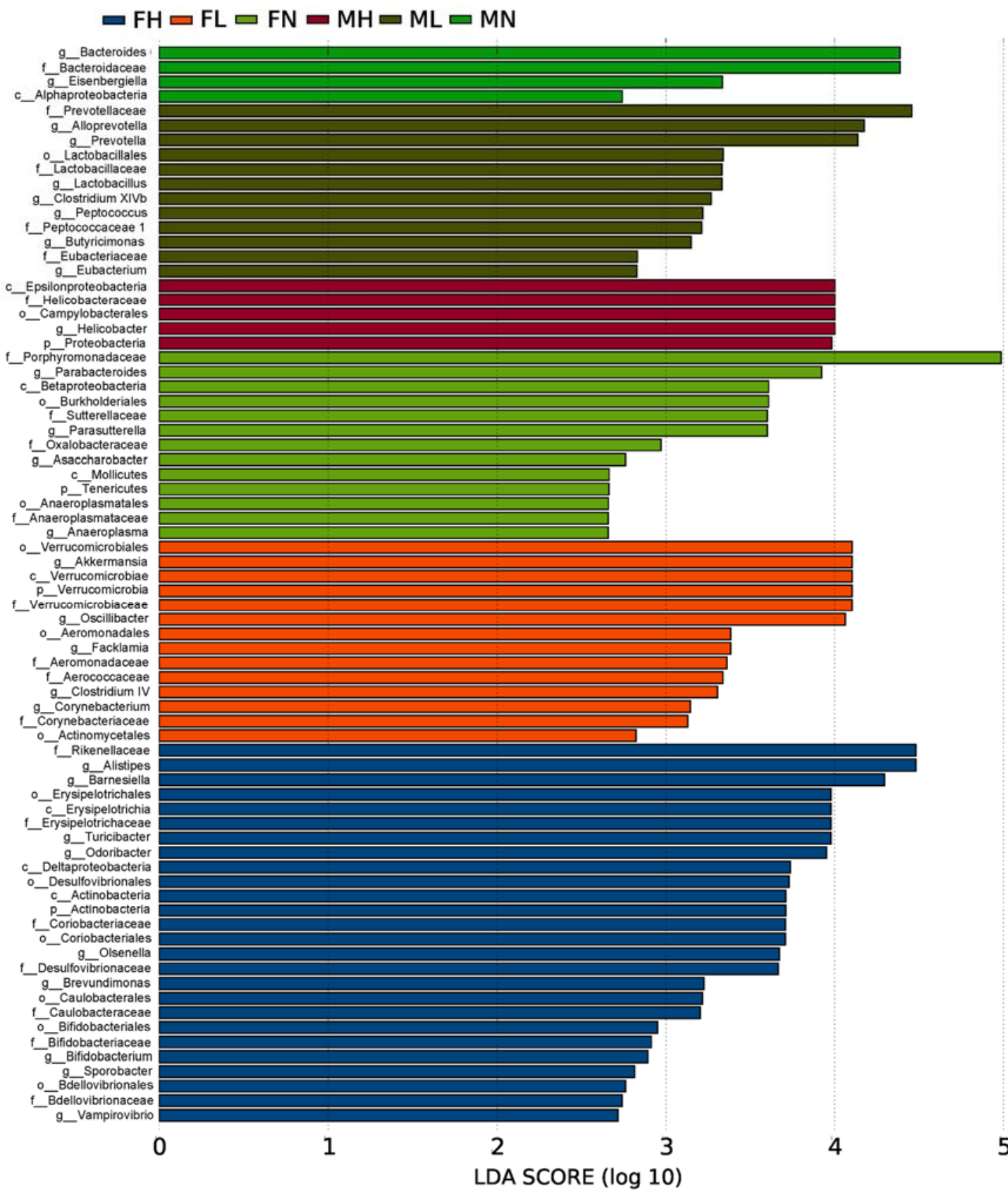

Figure 5. The LDA score obtained from LEfSe analysis of gut microbiota in different groups. A LDA effect size of more than 2 was used as a threshold for the LEfSe analysis.

It is of great interest to note that ECP remarkably increased the populations of Akkermansia muciniphila, Bifidobacterium spp. and Lactobacillus spp. in the gut (Figures 6). A. muciniphila is the only member of the genus Akkermansia spp. and the phylum Verrucomicrobia [40-42]. We and others have demonstrated that as an anti-inflammatory bacterium, A. muciniphila is inversely related to dysbiosis-associated diseases such as diabetes [43], obesity [44], metabolic syndrome [45] and colitis [46]. Recently, a large body of evidence has suggested the use of A. muciniphila for the treatment of metabolic disorders and gut diseases that are characterized with low-grade inflammation [40-42,45]. As such, A. muciniphila is now being widely developed as a next-generation probiotic and strategies to increase the abundance of A. muciniphila are currently a hot topic in this field [40,47]. Bifidobacterium spp. and Lactobacillus spp. are two common probiotics that have been extensively used in the food and pharmaceutical industries [48-50]. In addition, similar with that of A. muciniphila, Bifidobacterium spp. 
and Lactobacillus spp. have also been illustrated to be beneficial on a wide range of dysbiosis-associated diseases [51-55]. Here we demonstrate that ECP could significantly increase the abundances of A. muciniphila (by 3-fold), Bifidobacterium spp. (by 3-fold) and Lactobacillus spp. (by 35-fold) in C57BL/6J mice (Figure 6). However, it should be noted that ECP has a sex-specific effect on gut microbiota and, thus, the prebiotic effects on A. muciniphila and Bifidobacterium spp. were only found in female mice while that on Lactobacillus spp. were only found in male mice. Altogether, our data provides the first proof-of-concept for the use of ECP as a novel prebiotic for the promotion of health and management of dysbiosis-associated diseases.

A

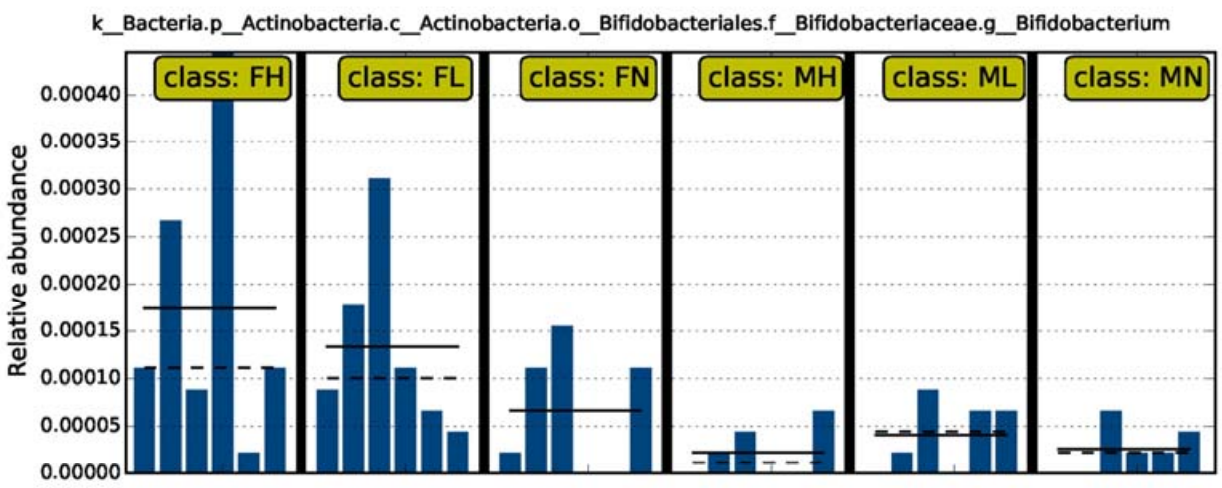

B

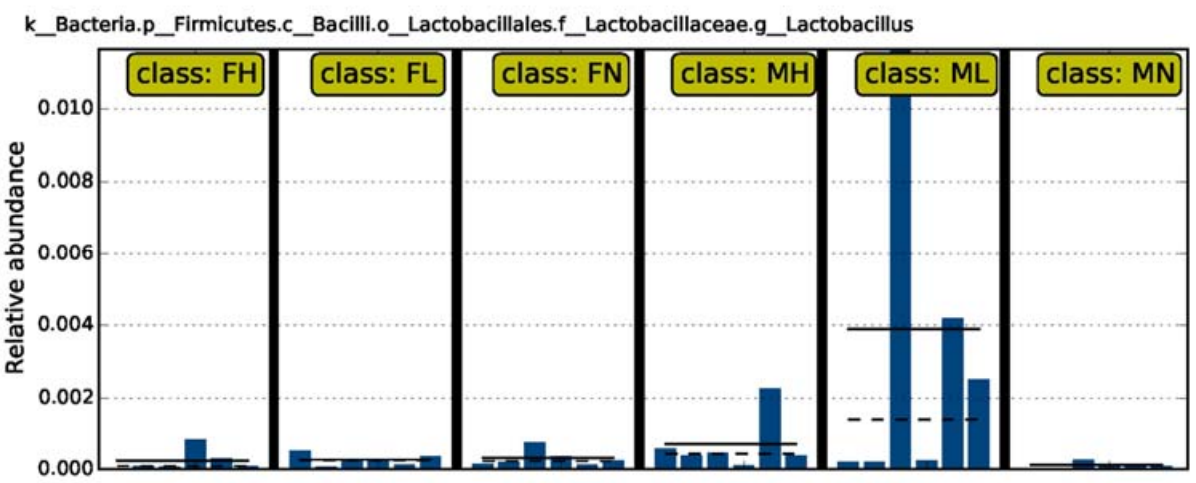

C

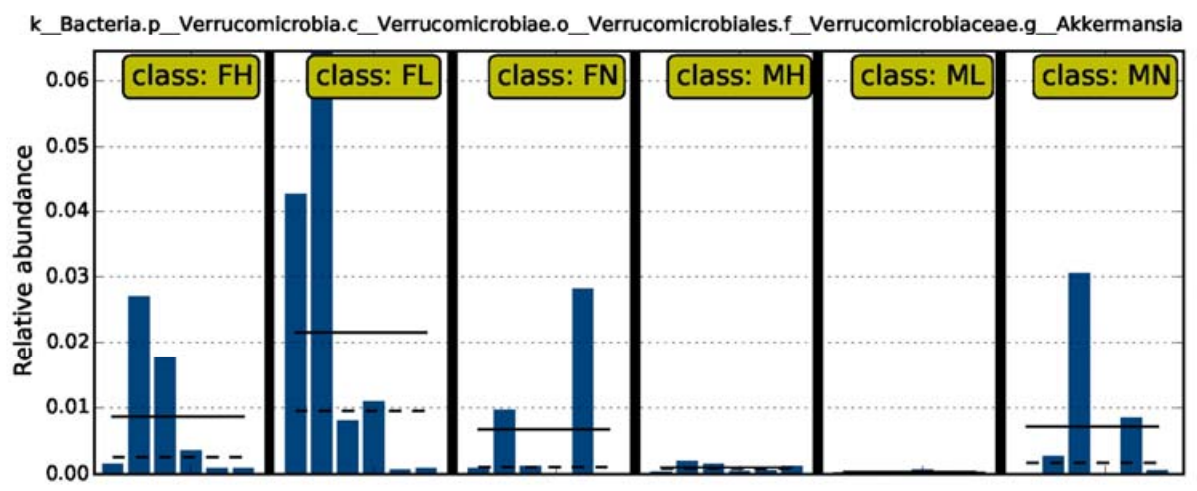

Figure 6. Intake of ECP significantly increased the abundances of Bifidobacterium spp. (A), Lactobacillus spp. (B) and A. muciniphila (C) in C57BL/6J mice. The solid line represents the average abundances of Bifidobacterium spp. (A), Lactobacillus spp. (B) and A. muciniphila (C) of the six replicates within each group; the dash line represents the median abundances of Bifidobacterium spp. (A), Lactobacillus spp. (B) and $A$. muciniphila $(\mathbf{C})$ of the six replicates within each group. The differences in the abundances of the probiotic bacteria between treated mice and control mice were evidenced to be significant at $p<0.05$ by LEfSe analysis. 
2.3. Dietary ECP Rduced the Antigen Load from the Gut in Female Mice and Maintained Normal Gut Homeostasis in Male Mice

Previous studies indicate that dietary fibers from marine seaweeds with a prebiotic effect could decrease the body weight of experimental mice $[29,56]$. In accordance with previous reports, ECP, a dietary fiber, was also found to decrease the body weight and energy intake in male and female mice (Figure 7). Given that elevated concentrations of SCFAs could promote satiety and regulate energy homeostasis, the body weight-reducing effect of ECP may be resulted from the fact that ECP stimulates the growth of SCFA-producing bacteria and increases intestinal levels of SCFAs in the gut.

A

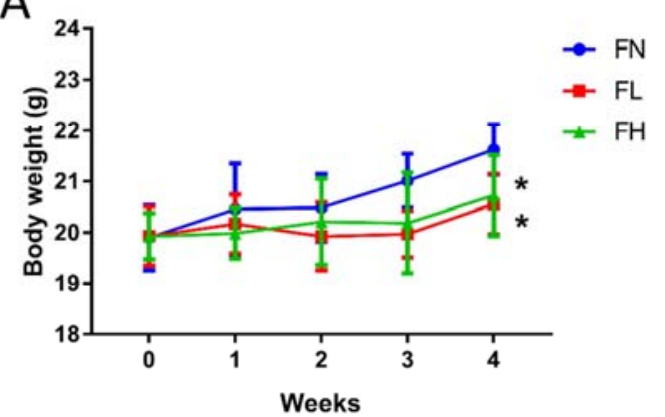

C

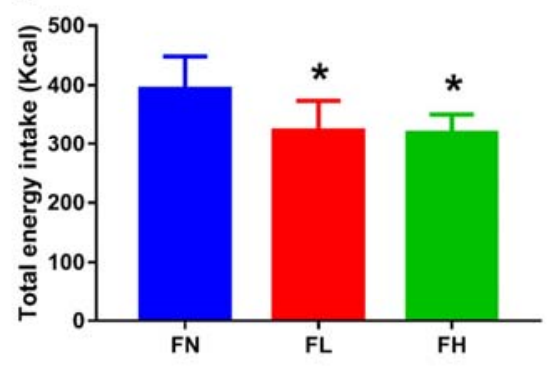

E

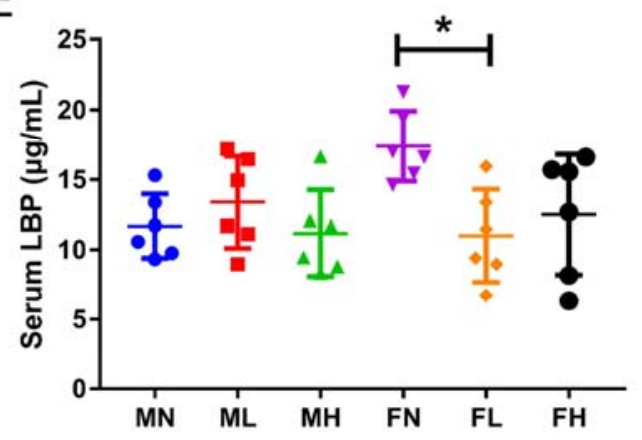

B

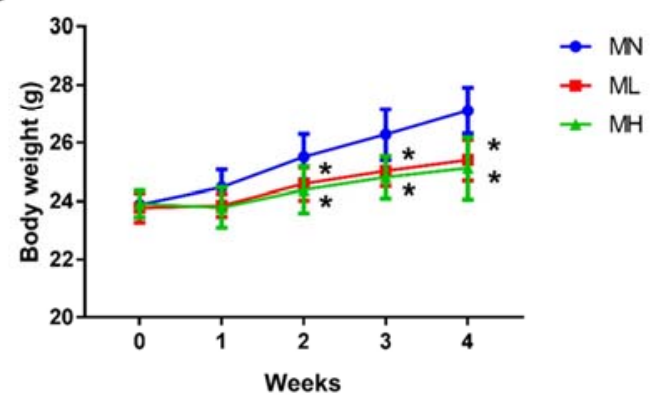

D
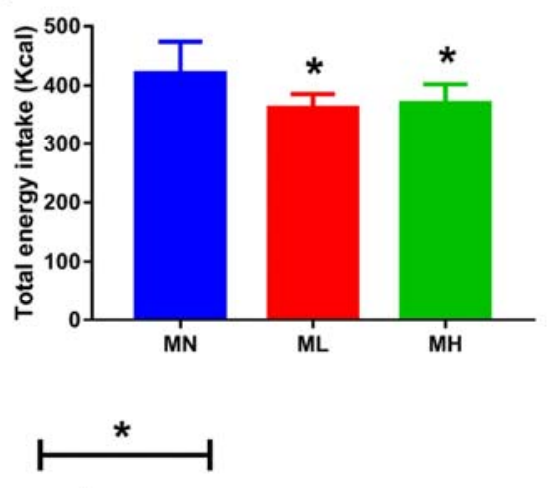
changes in serum LBP levels in male mice, suggesting that ECP had no toxic effect on the microbiome and could help to maintain normal gut homeostasis. As have detailed above, ECP has a differing effect on male and female microbiota. In this light, the discrepancy of serum LBP response to ECP treatment between male and female mice may be related to the sex-specific effect of ECP on gut microbiota.

Our study has two limitations. First, organ blood flow plays a pivotal role in maintaining the colonic mucosal integrity [59]. In addition, previous studies have demonstrated beneficial impacts of microcirculation on protection and healing of the mucosa in the gastrointestinal tract $[60,61]$. Ghrelin and obestatin are two important peptides with a multiplicity of physiological functions $[62,63]$. In the intestine, ghrelin and obestatin exert fundamental effects on colonic homeostasis, food intake, immune function and glucose and energy metabolism by regulating microcirculation [64-68]. In this sense, given that ECP significantly modulated the composition of the gut microbiota and reduced the food intake of C57BL/6J mice, it is highly possible that ECP could also have changed the serum levels of ghrelin and obestatin. However, due to the experimental design, we only focused on the effect of ECP on gut microbiota and as such, we did not determine the concentrations of these two bioactive peptides in the microcirculation system of the intestine. Therefore, we anticipate that future studies could provide more information on this topic. Second, it has been demonstrated that an increase of the probiotic bacteria, including A. muciniphila, Bifidobacterium spp. and Lactobacillus spp., could change the structure of the mucus layer in the colon [40-47,51-55]. However, as we primarily focused on the modulatory effect of ECP on the gut microbiome, we did not conduct any histological tests of the colon tissues. Therefore, further studies are encouraged to address this issue.

Altogether, our study demonstrates for the first time a favorable effect of ECP on gut microbiota by promoting the growth of beneficial microbes and forms the basis for the development of ECP as a novel prebiotic for health promotion and disease management.

\section{Materials and Methods}

\subsection{Chemicals and Reagents}

ECP was isolated and purified from Enteromorpha clathrata (sourced from Qingdao, China) and was obtained from Qingdao Seawin Biotech Group (Qingdao, China). The chemical structures of ECP were determined applying the protocols described elsewhere [45-56]. The molecular weight and sulfate content of ECP were found to be $11.67 \mathrm{kDa}$ and $14.7 \%$. The monosaccharide composition of ECP was determined as Man:Rha:GlcA:Glc:Gal:Xyl = 1.0\%:49.7\%:10.8\%:29.9\%:1.3\%:7.2\%. All other chemicals used were at analytical grade and were obtained from Sigma (Shanghai, China).

\subsection{Animals and Treatment}

All the animal experiments were approved by the Ethical Committee of Ocean University of China (Permission No. OUC-2017-0509-01). Briefly, a total of 36 specific pathogen-free C57BL/6J mice (six-week old, 18 males and 18 females) were purchased from the Vital River Laboratory Animal Technology Co. Ltd. (Beijing, China) (Certificate No. SCXK (Jing) 2016-0011). All mice were housed in a well-controlled environment $\left(22-23^{\circ} \mathrm{C}, 12 \mathrm{~h}\right.$ light/dark cycles). After a three-week adaptation period, all animals were randomly allocated into 6 test groups: male control group (MN), male low-dosage group (ML), male high-dosage group ( $\mathrm{MH})$, female control group (FN), female low-dosage group (FL) and female high-dosage group (FH). The low-dosage groups were given ECP at $50 \mathrm{mg} / \mathrm{kg} /$ day while the high-dosage groups were given ECP at $100 \mathrm{mg} / \mathrm{kg} /$ day. ECP was given by gavage and the control groups were given equal volume of normal saline. After 4 weeks of treatment, all mice of the 6 groups were sacrificed by cervical dislocation. Blood samples were collected from the orbital plexus and centrifuged at $1200 \mathrm{rpm}$ for $20 \mathrm{~min}$ to pellet the blood cells. The serum LBP levels were determined using a commercial ELISA kit (Cell Sciences, Canton, MA, USA). The cecum was harvested and the cecal content in each mouse was aseptically collected and stored at $-80^{\circ} \mathrm{C}$ before being analyzed. 


\subsection{DNA Preparation and $16 S$ rRNA High-throughput Sequencing}

The metagenomic DNA was extracted from the cecal content using a QIAamp DNA Stool Mini Kit (Qiagen, Hamburg, Germany). The concentrations of the obtained DNA were determined using a Nanodrop ND2000 UV-vis spectrophotometer (Thermo Scientific, Wilmington, NC USA). After checking the DNA quality by gel electrophoresis, a pair of universal primers $(341 \mathrm{~F}$ 5'-CCTACGGGRSGCAGCAG-3' and 806R 5'-GGACTACCAGGGTATCTAAT-3') were then applied to specifically amplify the V3-V4 hypervariable regions of the 16S rRNA gene using the method previously described $[45,56]$. The amplicons were purified and quantified before being sequenced in the HiSeq 2500 platform (Illumina, San Diego, CA USA) from a commercial company (Realbio Technology Co. Ltd., Shanghai, China).

\subsection{Bioinformatics and Sequencing Data Analysis}

USEARCH was applied to filter the chimeras and singletons of the raw sequencing data and to cluster the high-quality reads into operational taxonomic units (OTUs) at the $97 \%$ similarity level. After that, a representative sequence of each of the obtained OTUs was assigned to a taxonomic level in the Ribosomal Database Project (RDP) database using the RDP taxonomic nomenclature and the RDP classifier. To evaluate the community diversity and community richness, the values of Chao1, observed species and Shannon indices were calculated using Mothur (version V.1.30.1, University of Michigan, Ann Arbor, MI, USA). Principal component analysis (PCA) of the OTUs in different groups was conducted using R packages (version 3.1.0, R Foundation for Statistical Computing, Vienna, Austria) to compare the structure of the gut microbiota of male and female mice [45-56]. LEfSe analysis coupled with the Kruskal-Wallis rank sum test was performed to identify the microbial differences among all the groups [45-56]. The LDA score for each bacterium was calculated and a taxonomic cladogram was constructed to visualize the differences of the microbial composition. A significant value of less than 0.05 and a LDA effect size of more than 2 was used as thresholds for the LEfSe analysis.

\subsection{Statistical Analysis}

Data for Chao1, observed species, Shannon indices, body weight, total energy intake and serum LBP levels were expressed as mean \pm standard deviation. Statistical analyses of the above data were performed by one-way ANOVA followed by Dunnett's test (GraphPad Prism 6.00, La Jolla, CA, USA). All results were considered statistically significant at $p<0.05$ versus the control group.

Author Contributions: Q.S. and G.Y. conceived and designed the experiments; Q.S., Y.W., L.P., Q.N. and C.L. carried out the experiments; Q.S., Y.W. and L.P. analyzed the data; Q.S., Q.N. and C.L. interpreted the results; Q.S. wrote and revised the manuscript; H.J., C.C., J.H. and G.L. carefully reviewed and provided useful suggestions to improve the manuscript. G.Y. critically revised the manuscript and gave final approval of the version published.

Funding: This research was funded by [National Natural Science Foundation of China, NSFC] grant numbers [31670811 and 31600646], [NSFC-Shandong Joint Fund for Marine Science Research Centers] grant number [U1606403], [Major Science and Technology projects in Shandong province] grant number [2015ZDJS04002], [Fundamental Research Funds for the Central Universities] grant number [201762002], [Scientific and Technological Innovation Project Financially Supported by Qingdao National Laboratory for Marine Science and Technology] grant numbers [2015ASKJ02 and 2016ASKJ08-02] and [Taishan Scholar Project special funds] grant number [TS201511011].

Conflicts of Interest: The authors declare no conflict of interest.

\section{References}

1. Teng, Z.; Qian, L.; Zhou, Y. Hypolipidemic activity of the polysaccharides from Enteromorpha prolifera. Int. J. Biol. Macromol. 2013, 62, 254-256. [CrossRef] [PubMed]

2. Tang, Z.; Gao, H.; Wang, S.; Wen, S.; Qin, S. Hypolipidemic and antioxidant properties of a polysaccharide fraction from Enteromorpha prolifera. Int. J. Biol. Macromol. 2013, 58, 186-189. [CrossRef] [PubMed] 
3. Qi, X.; Mao, W.; Gao, Y.; Chen, Y.; Chen, Y.; Zhao, C.; Li, N.; Wang, C.; Yan, M.; Lin, C.; et al. Chemical characteristic of an anticoagulant-active sulfated polysaccharide from Enteromorpha clathrata. Carbohyd. Polym. 2012, 90, 1804-1810. [CrossRef] [PubMed]

4. Kim, J.K.; Cho, M.L.; Karnjanapratum, S.; Shin, I.S.; You, S.G. In vitro and in vivo immunomodulatory activity of sulfated polysaccharides from Enteromorpha prolifera. Int. J. Biol. Macromol. 2011, 49, 1051-1058. [CrossRef] [PubMed]

5. Pan, S.; Zhu, Q.; Wang, S.; Zang, Y. Enzymatic-assisted extraction process and antioxidant activity of polysaccharides from green alga Enteromorpha clathrata. Fish. Sci. 2013, 32, 187-191.

6. Cho, M.; Yang, C.; Kim, S.M.; You, S. Molecular characterization and biological activities of watersoluble sulfated polysaccharides from Enteromorpha prolifera. Food Sci. Biotechnol. 2010, 19, 525-533. [CrossRef]

7. Wang, L.; Wang, X.; Wu, H.; Liu, R. Overview on biological activities and molecular characteristics of sulfated polysaccharides from marine green algae in recent years. Mar. Drugs 2014, 12, 4984-5020. [CrossRef] [PubMed]

8. Jiao, G.; Yu, G.; Zhang, J.; Ewart, H.S. Chemical structures and bioactivities of sulfated polysaccharides from marine algae. Mar. Drugs 2011, 9, 196-223. [CrossRef] [PubMed]

9. Nicholson, J.K.; Holmes, E.; Kinross, J.; Burcelin, R.; Gibson, G.; Jia, W.; Pettersson, S. Host-gut microbiota metabolic interactions. Science 2012, 336, 1262-1267. [CrossRef] [PubMed]

10. Sommer, F.; Bäckhed, F. The gut microbiota-Masters of host development and physiology. Nat. Rev. Microbiol. 2013, 11, 227-238. [CrossRef] [PubMed]

11. Burcelin, R.; Serino, M.; Chabo, C.; Blasco-Baque, V.; Amar, J. Gut microbiota and diabetes: From pathogenesis to therapeutic perspective. Acta Diabetol. 2011, 48, 257-273. [CrossRef] [PubMed]

12. Cani, P.D.; Bibiloni, R.; Knauf, C.; Waget, A.; Neyrinck, A.M.; Delzenne, N.M.; Burcelin, R. Changes in gut microbiota control metabolic endotoxemia-induced inflammation in high-fat diet-induced obesity and diabetes in mice. Diabetes 2008, 57, 1470-1481. [CrossRef] [PubMed]

13. Jose, P.A.; Raj, D. Gut microbiota in hypertension. Curr. Opin. Nephrol. Hypertens. 2015, 24, 403. [CrossRef] [PubMed]

14. Louis, P.; Hold, G.L.; Flint, H.J. The gut microbiota, bacterial metabolites and colorectal cancer. Nat. Rev. Microbiol. 2014, 12, 661-672. [CrossRef] [PubMed]

15. Miyake, S.; Kim, S.; Suda, W.; Oshima, K.; Nakamura, M.; Matsuoka, T.; Chihara, N.; Tomita, A.; Sato, W.; Kim, S.W.; et al. Dysbiosis in the gut microbiota of patients with multiple sclerosis, with a striking depletion of species belonging to clostridia XIVA and IV clusters. PLoS ONE 2015, 10, e0137429. [CrossRef] [PubMed]

16. Abrahamsson, T.R.; Jakobsson, H.E.; Andersson, A.F.; Björkstén, B.; Engstrand, L.; Jenmalm, M.C. Low gut microbiota diversity in early infancy precedes asthma at school age. Clin. Exp. Allergy 2014, 44, 842-850. [CrossRef] [PubMed]

17. Li, Q.; Han, Y.; Dy, A.B.C.; Hagerman, R.J. The gut microbiota and autism spectrum disorders. Front. Cell. Neurosci. 2017, 11, 120. [CrossRef] [PubMed]

18. Hill, J.M.; Bhattacharjee, S.; Pogue, A.I.; Lukiw, W.J. The gastrointestinal tract microbiome and potential link to Alzheimer's disease. Front. Neurol. 2014, 5, 43. [CrossRef] [PubMed]

19. Mulak, A.; Bonaz, B. Brain-gut-microbiota axis in Parkinson's disease. World J. Gastroenterol. 2015, $21,10609$. [CrossRef] [PubMed]

20. Abu-Shanab, A.; Quigley, E.M.M. The role of the gut microbiota in nonalcoholic fatty liver disease. Nat. Rev. Gastroenterol. Hepatol. 2010, 7, 691-701. [CrossRef] [PubMed]

21. Jia, W.; Li, H.; Zhao, L.; Nicholson, J.K. Gut microbiota: A potential new territory for drug targeting. Nat. Rev. Drug Discov. 2008, 7, 123-129. [CrossRef] [PubMed]

22. Bindels, L.B.; Delzenne, N.M.; Cani, P.D.; Walter, J. Towards a more comprehensive concept for prebiotics. Nat. Rev. Gastroenterol. Hepatol. 2015, 12, 303-310. [CrossRef] [PubMed]

23. Gibson, G.R.; Hutkins, R.; Sanders, M.E.; Prescott, S.L.; Reimer, R.A.; Salminen, S.J.; Scott, K.; Stanton, C.; Swanson, K.S.; Cani, P.D.; et al. Expert consensus document: The International Scientific Association for Probiotics and Prebiotics (ISAPP) consensus statement on the definition and scope of prebiotics. Nat. Rev. Gastroenterol. Hepatol. 2017, 14, 491-502. [CrossRef] [PubMed] 
24. Dembiński, A.; Warzecha, Z.; Ceranowicz, P.; Dembiński, M.; Cieszkowski, J.; Gosiewski, T.; Bulanda, M.; Kuśnierz-Cabala, B.; Gałązka, K.; Konturek, P.C. Synergic interaction of rifaximin and mutaflor (Escherichia coli Nissle 1917) in the treatment of acetic acid-induced colitis in rats. Gastroenterol. Res. Pract. 2016, 2016, 3126280. [CrossRef] [PubMed]

25. De Jesus Raposo, M.F.; de Morais, A.M.M.B.; de Morais, R.M.S.C. Emergent sources of prebiotics: Seaweeds and microalgae. Mar. Drugs 2016, 14, 27. [CrossRef] [PubMed]

26. Shang, Q.; Jiang, H.; Cai, C.; Hao, J.; Li, G.; Yu, G. Gut microbiota fermentation of marine polysaccharides and its effects on intestinal ecology: An overview. Carbohyd. Polym. 2018, 179, 173-185. [CrossRef] [PubMed]

27. Kong, Q.; Dong, S.; Gao, J.; Jiang, C. In vitro fermentation of sulfated polysaccharides from E. prolifera and L. japonica by human fecal microbiota. Int. J. Biol. Macromol. 2016, 91, 867-871. [CrossRef] [PubMed]

28. Shang, Q.; Shi, J.; Song, G.; Zhang, M.; Cai, C.; Hao, J.; Li, G.; Yu, G. Structural modulation of gut microbiota by chondroitin sulfate and its oligosaccharide. Int. J. Biol. Macromol. 2016, 89, 489-498. [CrossRef] [PubMed]

29. Shang, Q.; Li, Q.; Zhang, M.; Song, G.; Shi, J.; Jiang, H.; Cai, C.; Hao, J.; Li, G.; Yu, G. Dietary keratan sulfate from shark cartilage modulates gut microbiota and increases the abundance of Lactobacillus spp. Mar. Drugs 2016, 14, 224. [CrossRef] [PubMed]

30. Markle, J.G.M.; Frank, D.N.; Mortin-Toth, S.; Robertson, C.E.; Feazel, L.M.; Rolle-Kampczyk, U.; Bergen, M.; McCoy, K.D.; Macpherson, A.J.; Danska, J.S. Sex differences in the gut microbiome drive hormone-dependent regulation of autoimmunity. Science 2013, 339, 1084-1088. [CrossRef] [PubMed]

31. Sonnenburg, E.D.; Smits, S.A.; Tikhonov, M.; Higginbottom, S.K.; Wingreen, N.S.; Sonnenburg, J.L. Diet-induced extinctions in the gut microbiota compound over generations. Nature 2016, 529, 212-215. [CrossRef] [PubMed]

32. Sonnenburg, E.D.; Sonnenburg, J.L. Starving our microbial self: The deleterious consequences of a diet deficient in microbiota-accessible carbohydrates. Cell Metab. 2014, 20, 779-786. [CrossRef] [PubMed]

33. Desai, M.S.; Seekatz, A.M.; Koropatkin, N.M.; Kamada, N.; Hickey, C.A.; Wolter, M.; Pudlo, N.A.; Kitamoto, S.; Terrapon, N.; Muller, A.; et al. A dietary fiber-deprived gut microbiota degrades the colonic mucus barrier and enhances pathogen susceptibility. Cell 2016, 167, 1339-1353. [CrossRef] [PubMed]

34. Den Besten, G.; van Eunen, K.; Groen, A.K.; Venema, K.; Reijngoud, D.J.; Bakker, B.M. The role of short-chain fatty acids in the interplay between diet, gut microbiota, and host energy metabolism. J. Lipid Res. 2013, 54, 2325-2340. [CrossRef] [PubMed]

35. Canfora, E.E.; Jocken, J.W.; Blaak, E.E. Short-chain fatty acids in control of body weight and insulin sensitivity. Nat. Rev. Endocrinol. 2015, 11, 577-591. [CrossRef] [PubMed]

36. Wong, J.M.; de Souza, R.; Kendall, C.W.; Emam, A.; Jenkins, D.J. Colonic health: Fermentation and short chain fatty acids. J. Clin. Gastroenterol. 2006, 40, 235-243. [CrossRef] [PubMed]

37. Ríos-Covián, D.; Ruas-Madiedo, P.; Margolles, A.; Gueimonde, M.; de los Reyes-Gavilán, C.G.; Salazar, N. Intestinal short chain fatty acids and their link with diet and human health. Front. Microbiol. 2016, 7, 185. [CrossRef] [PubMed]

38. Kasubuchi, M.; Hasegawa, S.; Hiramatsu, T.; Ichimura, A.; Kimura, I. Dietary gut microbial metabolites, short-chain fatty acids, and host metabolic regulation. Nutrients 2015, 7, 2839-2849. [CrossRef] [PubMed]

39. Byrne, C.S.; Chambers, E.S.; Morrison, D.J.; Frost, G. The role of short chain fatty acids in appetite regulation and energy homeostasis. Int. J. Obes. 2015, 39, 1331-1338. [CrossRef] [PubMed]

40. Cani, P.D.; de Vos, W.M. Next-Generation Beneficial Microbes: The Case of Akkermansia muciniphila. Front. Microbiol. 2017, 8, 1765. [CrossRef] [PubMed]

41. Belzer, C.; de Vos, W.M. Microbes inside-from diversity to function: The case of Akkermansia. ISME J. 2012, 6, 1449-1458. [CrossRef] [PubMed]

42. Derrien, M.; Belzer, C.; de Vos, W.M. Akkermansia muciniphila and its role in regulating host functions. Microb. Pathog. 2017, 106, 171-181. [CrossRef] [PubMed]

43. Everard, A.; Belzer, C.; Geurts, L.; Ouwerkerk, J.P.; Druart, C.; Bindels, L.B.; Guiot, Y.; Derrien, M.; Muccioli, G.G.; Delzenne, N.M.; et al. Cross-talk between Akkermansia muciniphila and intestinal epithelium controls diet-induced obesity. Proc. Natl. Acad. Sci. USA 2013, 110, 9066-9071. [CrossRef] [PubMed]

44. Dao, M.C.; Everard, A.; Aron-Wisnewsky, J.; Sokolovska, N.; Prifti, E.; Verger, E.O.; Kayser, B.D.; Levenez, F.; Chilloux, J.; Hoyles, L.; et al. Akkermansia muciniphila and improved metabolic health during a dietary intervention in obesity: Relationship with gut microbiome richness and ecology. Gut 2016, 65, 426-436. [CrossRef] [PubMed] 
45. Shang, Q.; Song, G.; Zhang, M.; Shi, J.; Xu, C.; Hao, J.; Li, G.; Yu, G. Dietary fucoidan improves metabolic syndrome in association with increased Akkermansia population in the gut microbiota of high-fat diet-fed mice. J. Funct. Foods 2017, 28, 138-146. [CrossRef]

46. Shang, Q.; Sun, W.; Shan, X.; Jiang, H.; Cai, C.; Hao, J.; Li, G.; Yu, G. Carrageenan-induced colitis is associated with decreased population of anti-inflammatory bacterium, Akkermansia muciniphila, in the gut microbiota of C57BL/6J mice. Toxicol. Lett. 2017, 279, 87-95. [CrossRef] [PubMed]

47. Zhou, K. Strategies to promote abundance of Akkermansia muciniphila, an emerging probiotics in the gut, evidence from dietary intervention studies. J. Funct. Foods 2017, 33, 194-201. [CrossRef]

48. Gomes, A.M.; Malcata, F.X. Bifidobacterium spp. and Lactobacillus acidophilus: Biological, biochemical, technological and therapeutical properties relevant for use as probiotics. Trends Food Sci. Technol. 1999, 10, 139-157. [CrossRef]

49. Soccol, C.R.; de Souza Vandenberghe, L.P.; Spier, M.R.; Medeiros, A.B.P.; Yamaguishi, C.T.; De Dea Lindner, J.; Pandey, A.; Thomaz-Soccol, V. The potential of probiotics: A review. Food Technol. Biotechnol. 2010, 48, 413-434.

50. Saarela, M.; Mogensen, G.; Fonden, R.; Mättö, J.; Mattila-Sandholm, T. Probiotic bacteria: Safety, functional and technological properties. J. Biotechnol. 2000, 84, 197-215. [CrossRef]

51. Wang, J.; Tang, H.; Zhang, C.; Zhao, Y.; Derrien, M.; Rocher, E.; van Hylckama Vlieg, J.E.; Strissel, K.; Zhao, L.; Obin, M.; et al. Modulation of gut microbiota during probiotic-mediated attenuation of metabolic syndrome in high fat diet-fed mice. ISME J. 2015, 9, 1-15. [CrossRef] [PubMed]

52. Kim, S.W.; Park, K.Y.; Kim, B.; Kim, E.; Hyun, C.K. Lactobacillus rhamnosus GG improves insulin sensitivity and reduces adiposity in high-fat diet-fed mice through enhancement of adiponectin production. Biochem. Biophys. Res. Commun. 2013, 431, 258-263. [CrossRef] [PubMed]

53. Chen, J.; Wang, R.; Li, X.F.; Wang, R.L. Bifidobacterium adolescentis supplementation ameliorates visceral fat accumulation and insulin sensitivity in an experimental model of the metabolic syndrome. Brit. J. Nutr. 2012, 107, 1429-1434. [CrossRef] [PubMed]

54. Srutkova, D.; Schwarzer, M.; Hudcovic, T.; Zakostelska, Z.; Drab, V.; Spanova, A.; Rittich, B.; Kozakova, H.; Schabussova, I. Bifidobacterium longum CCM 7952 promotes epithelial barrier function and prevents acute DSS-induced colitis in strictly strain-specific manner. PLoS ONE 2015, 10, e0134050. [CrossRef] [PubMed]

55. Lye, H.S.; Kuan, C.Y.; Ewe, J.A.; Fung, W.Y.; Liong, M.T. The improvement of hypertension by probiotics: Effects on cholesterol, diabetes, renin, and phytoestrogens. Int. J. Mol. Sci. 2009, 10, 3755-3775. [CrossRef] [PubMed]

56. Shang, Q.; Shan, X.; Cai, C.; Hao, J.; Li, G.; Yu, G. Dietary fucoidan modulates the gut microbiota in mice by increasing the abundance of Lactobacillus and Ruminococcaceae. Food Funct. 2016, 7, 3224-3232. [CrossRef] [PubMed]

57. Zhang, C.; Li, S.; Yang, L.; Huang, P.; Li, W.; Wang, S.; Zhao, G.; Zhang, M.; Pang, X.; Yan, Z.; et al. Structural modulation of gut microbiota in life-long calorie-restricted mice. Nat. Commun. 2013, 4, 2163. [CrossRef] [PubMed]

58. Jaworek, J.; Tudek, B.; Kowalczyk, P.; Kot, M.; Szklarczyk, J.; Leja-Szpak, A.; Pierzchalski, P.; Bonior, J.; Dembiński, A.; Ceranowicz, P.; et al. Effect of endotoxemia in suckling rats on pancreatic integrity and exocrine function in adults: A review report. Gastroenterol. Res. Pract. 2018, 2018, 6915059. [CrossRef] [PubMed]

59. Leung, F.W.; Su, K.C.; Pique, J.M.; Thiefin, G.; Passaro, E.; Guth, P.H. Superior mesenteric artery is more important than inferior mesenteric artery in maintaining colonic mucosal perfusion and integrity in rats. Digest. Dis. Sci. 1992, 37, 1329-1335. [CrossRef] [PubMed]

60. Matuszyk, A.; Ceranowicz, D.; Warzecha, Z.; Ceranowicz, P.; Fyderek, K.; Gałązka, K.; Cieszkowski, J.; Bonior, J.; Jaworek, J.; Pihut, M.; et al. The influence of ghrelin on the development of dextran sodium sulfate-induced colitis in rats. Biomed. Res. Int. 2015, 2015, 718314. [CrossRef] [PubMed]

61. Matuszyk, A.; Ceranowicz, P.; Warzecha, Z.; Cieszkowski, J.; Bonior, J.; Jaworek, J.; Kuśnierz-Cabala, B.; Konturek, P.; Ambroży, T.; Dembiński, A. Obestatin accelerates the healing of acetic acid-induced colitis in rats. Oxidative Med. Cell. Longev. 2016, 2016, 2834386. [CrossRef] [PubMed]

62. Klok, M.D.; Jakobsdottir, S.; Drent, M.L. The role of leptin and ghrelin in the regulation of food intake and body weight in humans: A review. Obes. Rev. 2007, 8, 21-34. [CrossRef] [PubMed]

63. Soares, J.B.; Leite-Moreira, A.F. Ghrelin, des-acyl ghrelin and obestatin: Three pieces of the same puzzle. Peptides 2008, 29, 1255-1270. [CrossRef] [PubMed] 
64. Dembinski, A.; Warzecha, Z.; Ceranowicz, P.; Konturek, P.C.; Tomaszewska, R.; Stachura, J.; Konturek, S.J. Ghrelin attenuates the development of acute pancreatitis in rat. J. Physiol. Pharmacol. 2003, 54, 561-574. [PubMed]

65. Bukowczan, J.; Cieszkowski, J.; Warzecha, Z.; Ceranowicz, P.; Kusnierz-Cabala, B.; Tomaszewska, R.; Dembinski, A. Therapeutic effect of obestatin in the course of cerulein-induced acute pancreatitis. Pancreas 2016, 45, 700-706. [CrossRef] [PubMed]

66. Bukowczan, J.; Warzecha, Z.; Ceranowicz, P.; Kuśnierz-Cabala, B.; Tomaszewska, R. Obestatin accelerates the recovery in the course of ischemia/reperfusion-induced acute pancreatitis in rats. PLOS ONE 2015, 10, e0134380. [CrossRef] [PubMed]

67. Bukowczan, J.; Warzecha, Z.; Ceranowicz, P.; Kuśnierz-Cabala, B.; Tomaszewska, R.; Dembinski, A. Pretreatment with obestatin reduces the severity of ischemia/reperfusion-induced acute pancreatitis in rats. Eur. J. Pharmacol. 2015, 760, 113-121. [CrossRef] [PubMed]

68. Ceranowicz, D.; Warzecha, Z.; Dembinski, A.; Ceranowicz, P.; Cieszkowski, J.; Kusnierz-Cabala, B.; Kuwahara, A.; Kato, I. Role of hormonal axis, growth hormone-IGF-1, in therapeutic effect of ghrelin in the course of cerulein-induced acute pancreatitis. J. Physiol. Pharmacol. 2010, 61, 599-606. [PubMed]

(C) 2018 by the authors. Licensee MDPI, Basel, Switzerland. This article is an open access article distributed under the terms and conditions of the Creative Commons Attribution (CC BY) license (http:/ / creativecommons.org/licenses/by/4.0/). 